\title{
Transient Photo-Inactivation of Cell Membrane Protein Activity without Genetic Modification by Molecular Hyperthermia
}

Peiyuan Kang, ${ }^{\dagger}$ Xiaoqing Li, ${ }^{\dagger}$ Yaning Liu, ${ }^{\dagger}$ Stephanie I. Shiers, ${ }^{\S}$ Hejian Xiong, ${ }^{\dagger}$ Monica Giannotta," Elisabetta Dejana,,$\| \perp$ Theodore J. Price, ${ }^{\S}$ Jaona Randrianalisoa, ${ }^{\#}$ Steven O. Nielsen, ${ }^{\circ}$ Zhenpeng Qin*,†,०,

'Department of Mechanical Engineering, University of Texas at Dallas 800 West Campbell Rd., Richardson, Texas 75080, USA.

${ }^{\ddagger}$ Department of Bioengineering, University of Texas at Dallas

800 West Campbell Rd., Richardson, Texas 75080, USA.

${ }^{\S}$ School of Behavioral and Brain Sciences, University of Texas at Dallas

800 West Campbell Rd., Richardson, Texas 75080, USA.

"Vascular Biology Laboratory, The FIRC Institute of Molecular Oncology (IFOM), 20139 Milan, Italy.

${ }^{\perp}$ Department of Immunology, Genetics and Pathology, University of Uppsala 75105 Uppsala, Sweden.

"Institut de Thermique, Mécanique, Matériaux (ITheMM EA 7548), University of Reims Champagne-Ardenne, Reims Cedex 2, 51687 France

${ }^{\nabla}$ Department of Chemistry and Biochemistry, University of Texas at Dallas

800 West Campbell Rd., Richardson, Texas 75080, USA.

${ }^{\circ}$ Department of Surgery, University of Texas at Southwestern Medical Center

5323 Harry Hines Blvd., Dallas, Texas 75390, USA. 

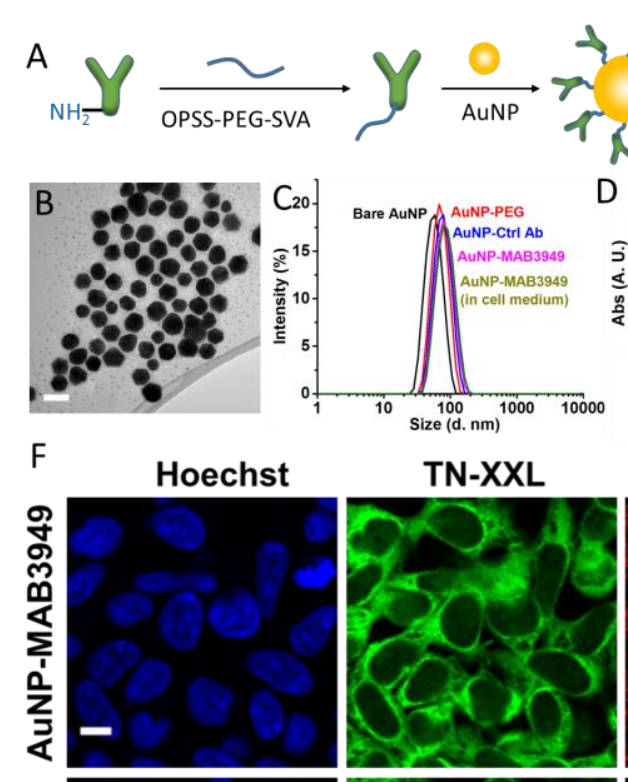

TN-XXL
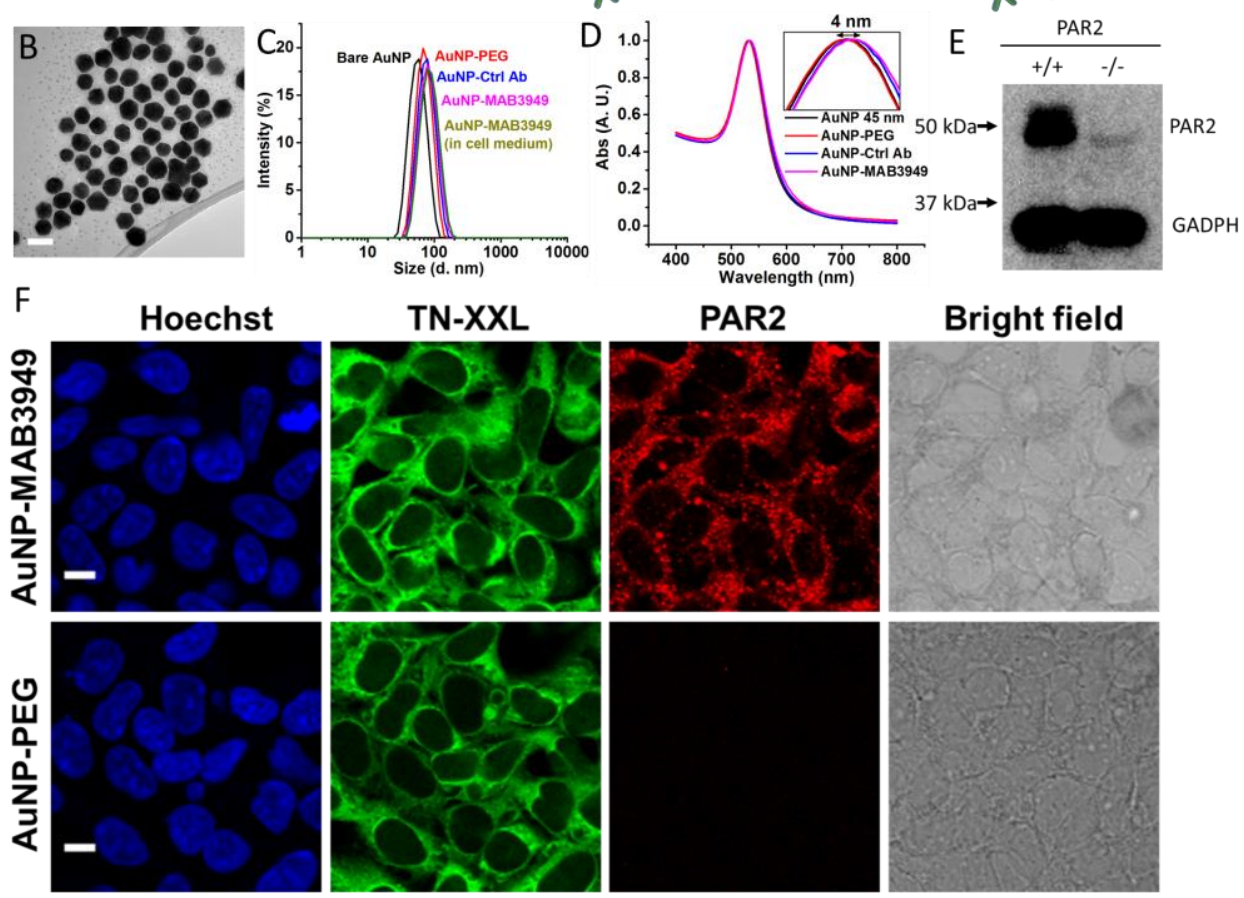

Bright field
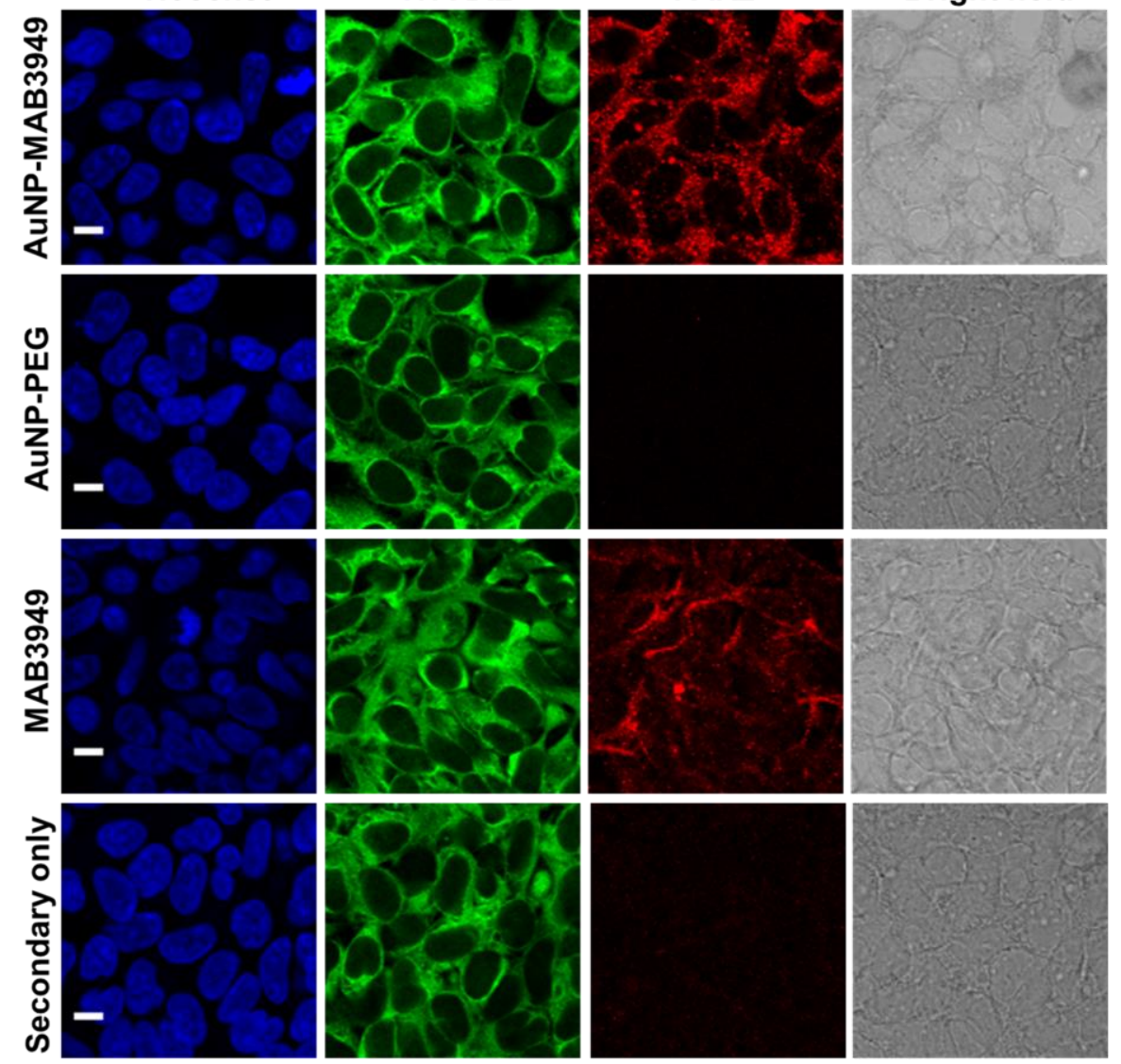

Figure S1. Characterization of PAR2-targeting plasmonic gold nanoparticles (AuNP). A) Schematic of the antibody functionalization on AuNP. B) Transmission electron microscopy (TEM) imaging for $45 \mathrm{~nm}$ AuNPs (Scale bar: $50 \mathrm{~nm}$ ). C) Dynamic light scattering (DLS) measurements for bare AuNP, polyethylene glycol (PEG) coated AuNP (AuNP-PEG), control antibody-coated AuNP (AuNP-Ctrl Ab), PAR2-targeting antibody-coated AuNP in borate buffer and in cell medium (AuNP-MAB3949). D) UV-Vis spectroscopy measurements of AuNPs before and after PEG and MAB3949 modification. The insert shows that the antibody conjugation shifts the peak by $4 \mathrm{~nm}$, from $532 \mathrm{~nm}$ to $536 \mathrm{~nm}$. E) Western blot of dorsal root ganglion (DRG) samples from wild type mouse (PAR2 +/+) and PAR2 knockout mouse (PAR2 -/-), GADPH refers to Glyceraldehyde 3-phosphate dehydrogenase. F) Fluorescent immunocytochemistry imaging of HEK293 cells (prefixed with methanol). Nucleus is in blue (Hoechst), FRET $\mathrm{Ca}^{2+}$ indicator (TN-XXL) is in green, and PAR2 is in red (Alexa 633). AuNP-MAB3949, AuNP-PEG, MAB3949 groups represent incubating PAR2 antibody-AuNP conjugate, AuNP-PEG conjugate, and free PAR2 antibody (MAB3949( with the cell after methanol fixing, respectively. The secondary only refers to incubating the cell with secondary antibody only without the primary PAR2 antibody. Scale bar: $10 \mu \mathrm{m}$. 


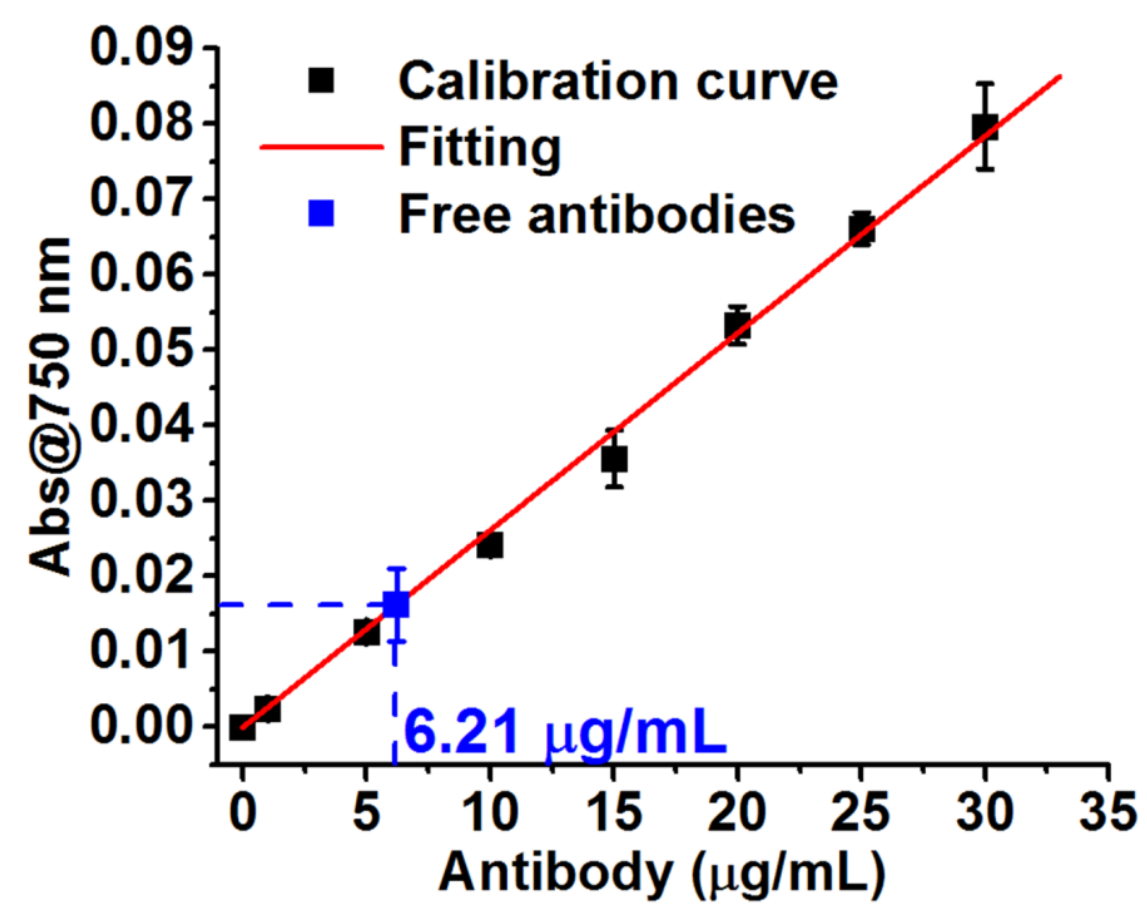

Figure S2. The standard calibration curve to determine antibody concentration on the AuNP surface. Protein concentration on the AuNP surface was determined by measuring the free antibody in the supernatant after centrifugations $(6.21 \mu \mathrm{g} / \mathrm{mL})$. 

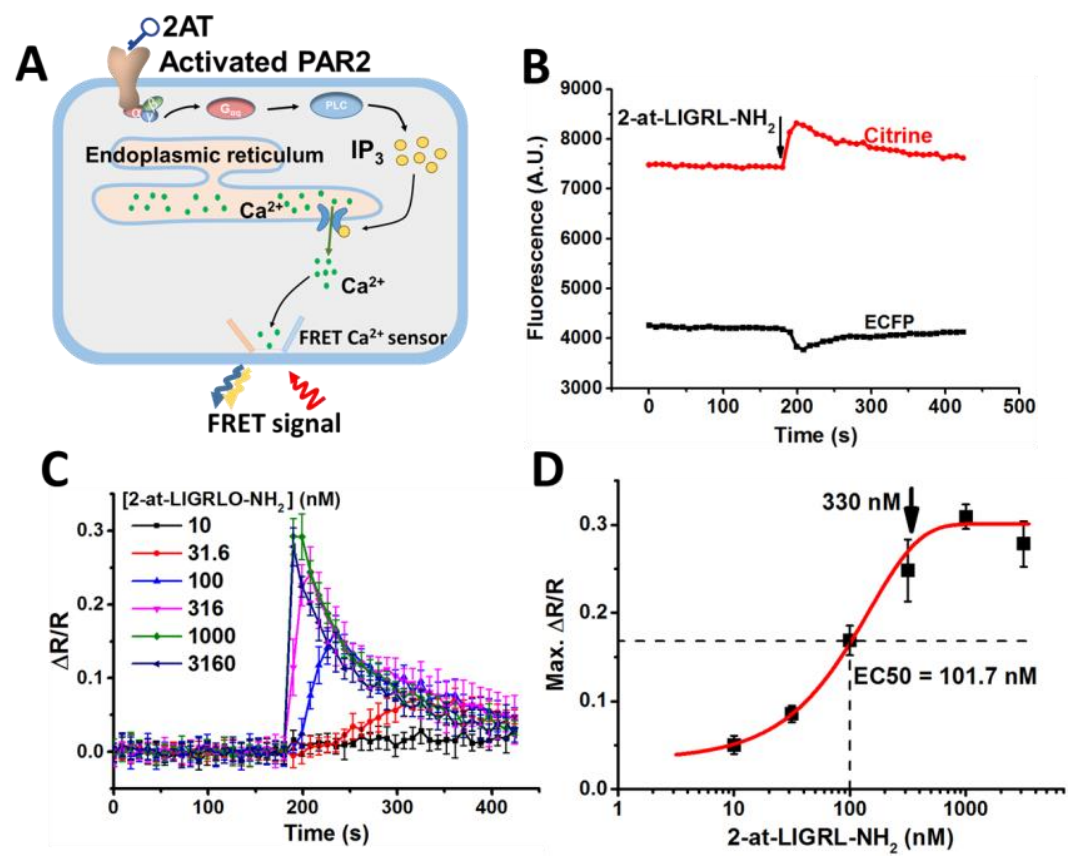

Figure S3. $\mathrm{Ca}^{2+}$ signaling induced by PAR2 activation. A) Schematic illustrating $\mathrm{Ca}^{2+}$ signaling due to PAR2 activation. PAR2 agonist 2-at-LIGRL-NH2 (2AT) binding leads to robust $\mathrm{Ca}^{2+}$ signals that can be measured by a FRET-based $\mathrm{Ca}^{2+}$ sensor (TN-XXL). B) FRET response of $\mathrm{Ca}^{2+}$ release due to PAR2 activation by $2 \mathrm{AT}(316 \mathrm{nM})$. C) The FRET ratio $(\Delta \mathrm{R} / \mathrm{R})$ signal for different concentrations of 2AT. D) Doseresponse curve of maximum $\Delta \mathrm{R} / \mathrm{R}$ for HEK293 cell in response to $2 \mathrm{AT}(\mathrm{EC} 50=101.5 \pm 28.7 \mathrm{nM}, \mathrm{n}=4)$. The black arrow indicates the concentration of $2 \mathrm{AT}$ used in this study. 


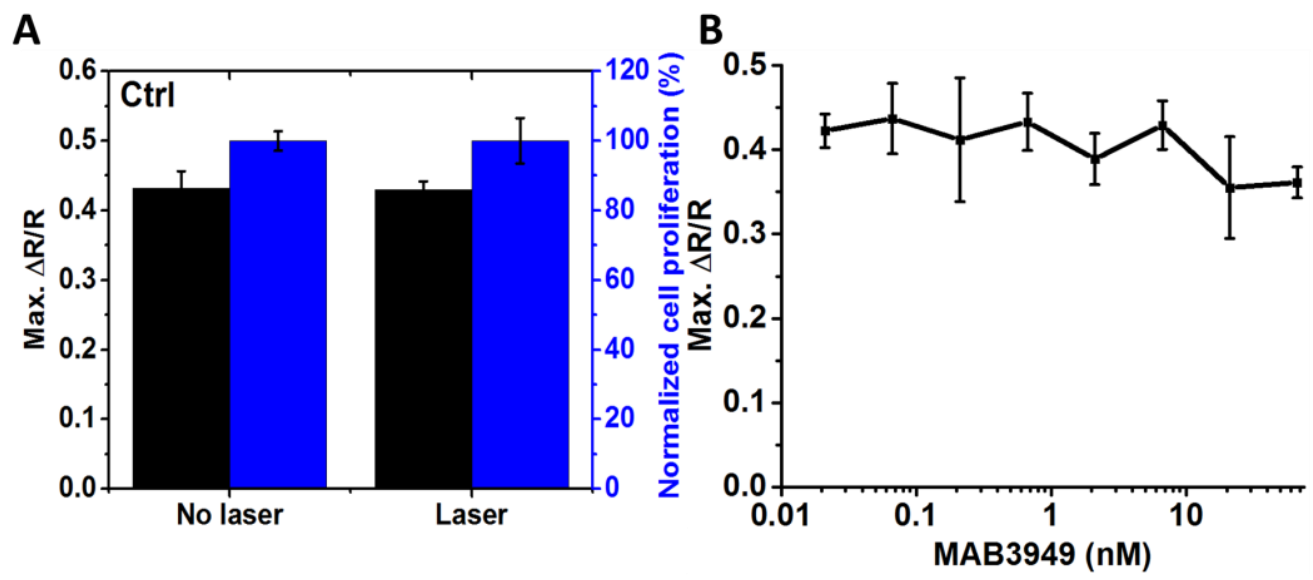

Figure S4. Additional control experiments for molecular hyperthermia. A) PAR2 activity and cell viability for the control group (cells without AuNP incubation) before and 30 minutes after laser irradiation $\left(100 \mathrm{~mJ} / \mathrm{cm}^{2}, 10\right.$ pulses). B) PAR2 activity with MAB3949 incubation shows that MAB3949 alone does not affect PAR2 activity up to $66 \mathrm{nM}$. 

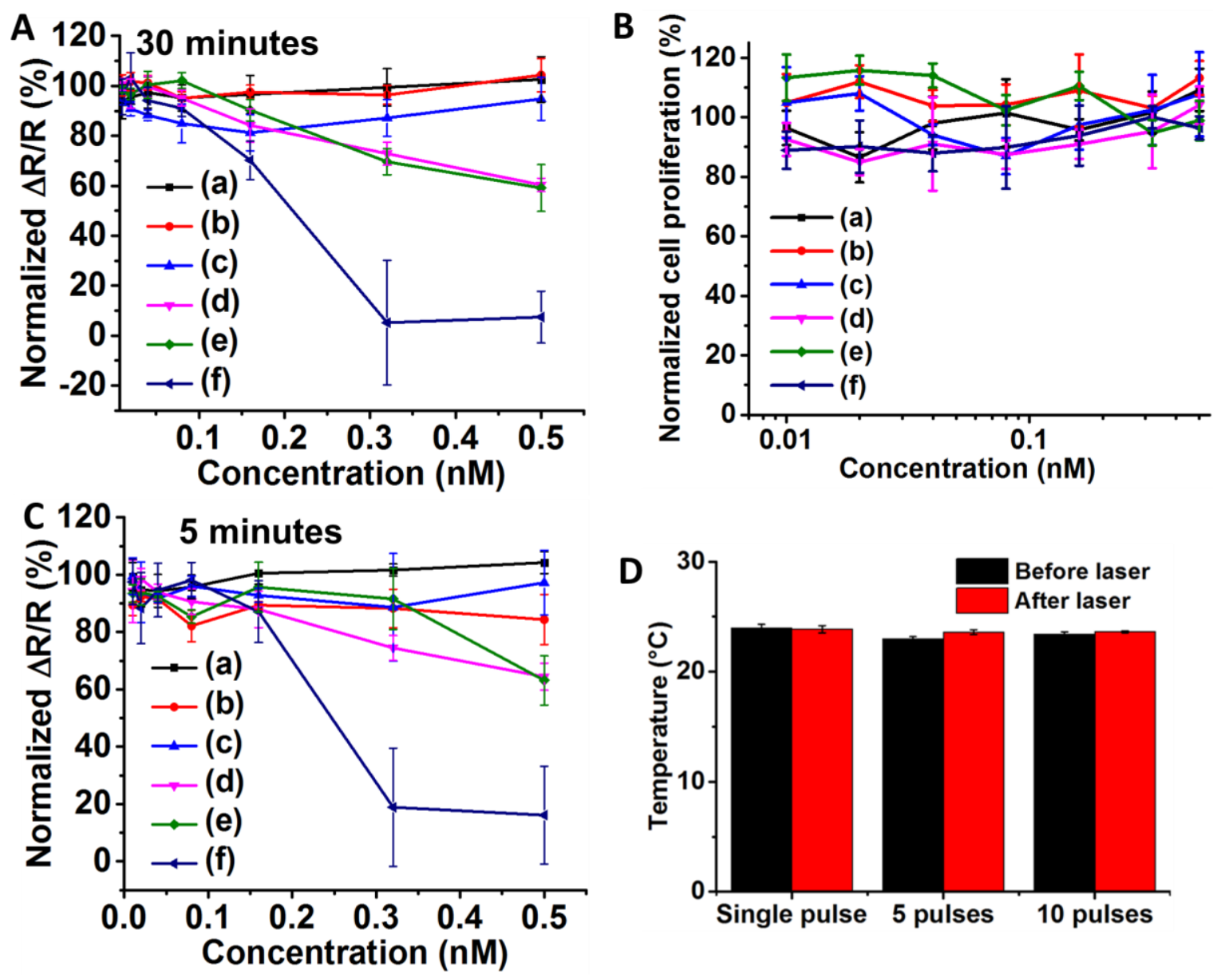

Figure S5. Additional data for A) Molecular hyperthermia of PAR2 under different particle concentrations. B) Cell proliferation for different groups. C) Replot of figure 2E by adding PEG coated AuNP. For all figures: (a) AuNP-PEG, no laser; (b) AuNP-control antibody (Ctrl Ab), no laser; (c) AuNP-MAB3949, no laser; (d) AuNP-PEG, with laser; (e) AuNP-Ctrl Ab, with laser; (f) AuNP-MAB3949, with laser. D) Bulk medium temperature measurement before and after laser, $[\mathrm{AuNP}]=0.5 \mathrm{nM}$. 


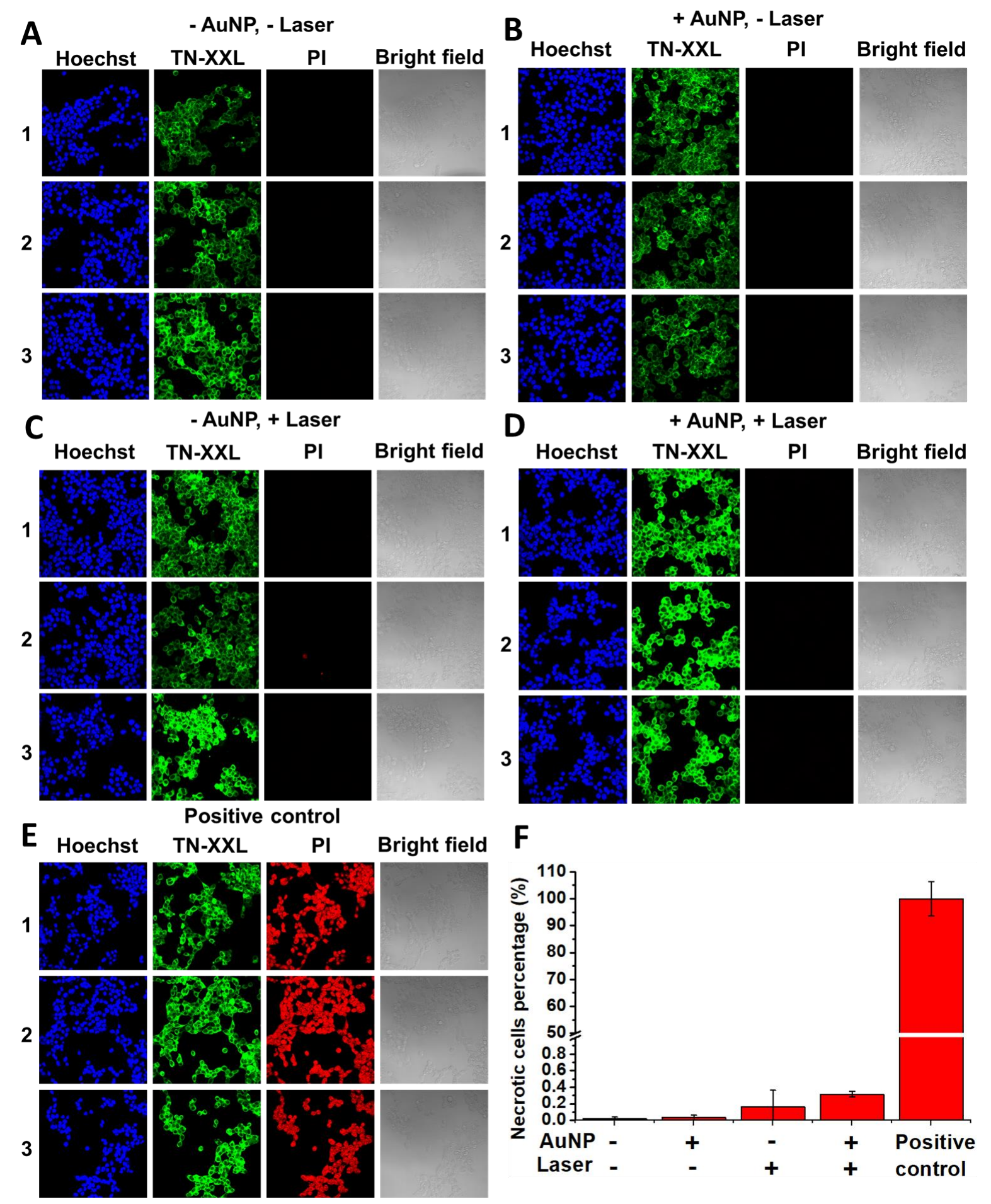

Figure S6. Propidium iodide (PI) staining assay to examine cell membrane integrity and necrosis. A) Negative control (no AuNP, no laser), B) With AuNP incubation, no laser irradiation. C) With laser irradiation (100 mJ/ $\mathrm{cm}^{2}, 10$ pulses), no AuNP incubation. D) MH group ([AuNP-MAB3949] $=0.32 \mathrm{nM}, 100$ $\mathrm{mJ} / \mathrm{cm}^{2}, 10$ pulses). E) Positive control (prefixed cells with 100\% methanol). F) Quantification of necrotic cells percentage. The percentage was calculated by number ratio of PI-stained and Hoechst-stained cells. 

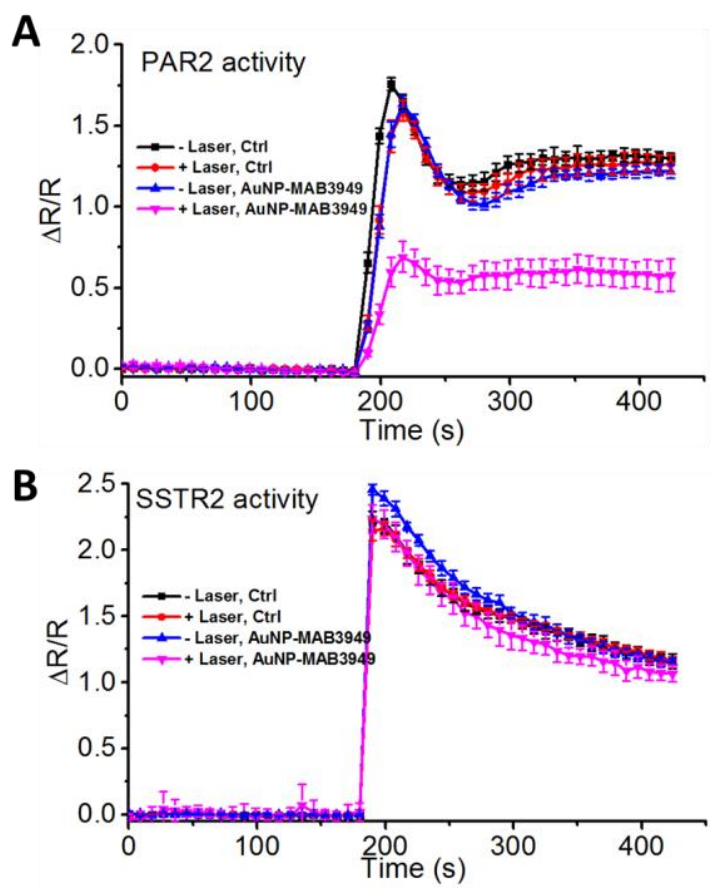

Figure S7. Selective photo-inactivation of protease activated receptor 2 (PAR2) without compromising somatostatin receptor 2 (SSTR2) activity. A) $\mathrm{Ca}^{2+}$ signal by PAR2 activation. $\mathrm{B}$ ) $\mathrm{Ca}^{2+}$ signal by SSTR2 activation. 

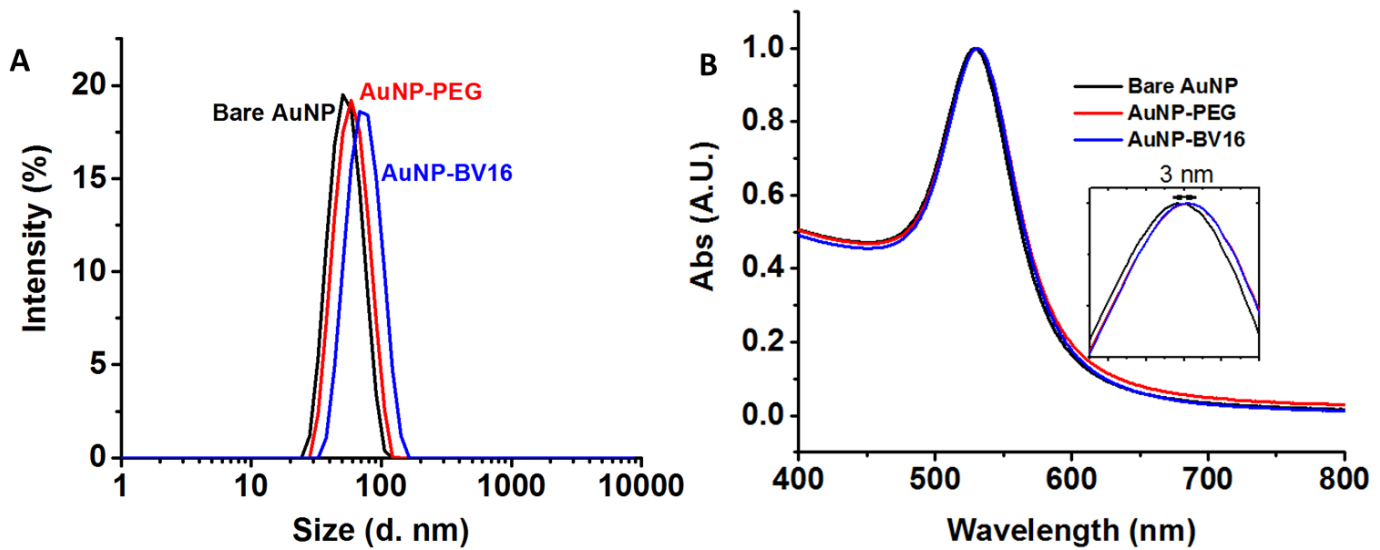

Figure S8. Characterization of JAM-A antibody (BV16) coated AuNP. A) DLS and B) UV-Vis measurements. AuNP-PEG is included as a control group. The insert shows that the antibody conjugation shifted the peak by $3 \mathrm{~nm}$. 


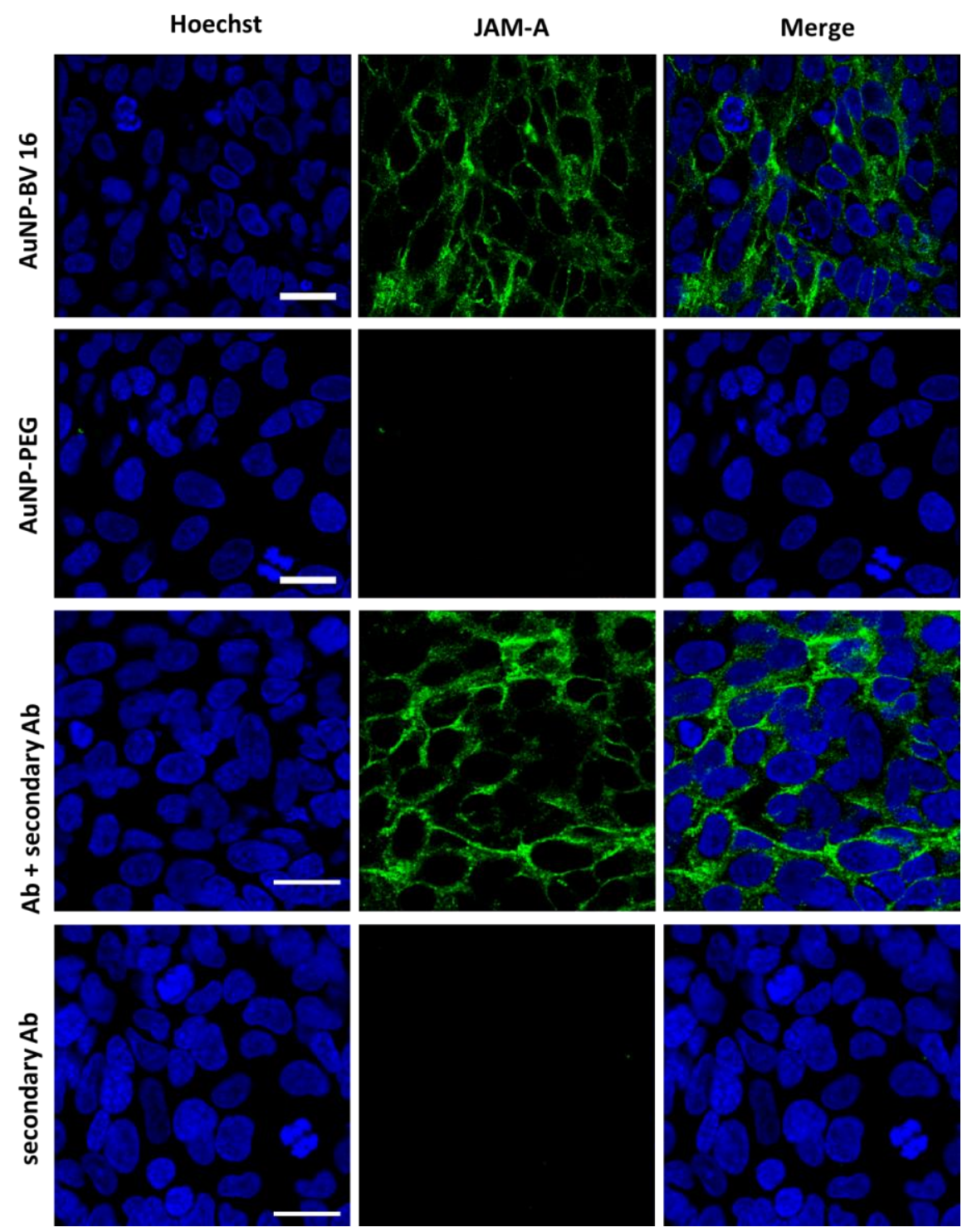

Figure S9. Fluorescent immunocytochemistry staining of hCMEC/D3 cells. AuNP concentration is 0.5 $\mathrm{nM}, \mathrm{BV}-16$ concentration is $5 \mu \mathrm{g} / \mathrm{mL}$ and incubation time is 30 mins. Nucleus is in blue (Hoechst), JAM-A is in green (secondary antibody conjugate with Alexa 488) (scale bar: $20 \mu \mathrm{m}$ ). 

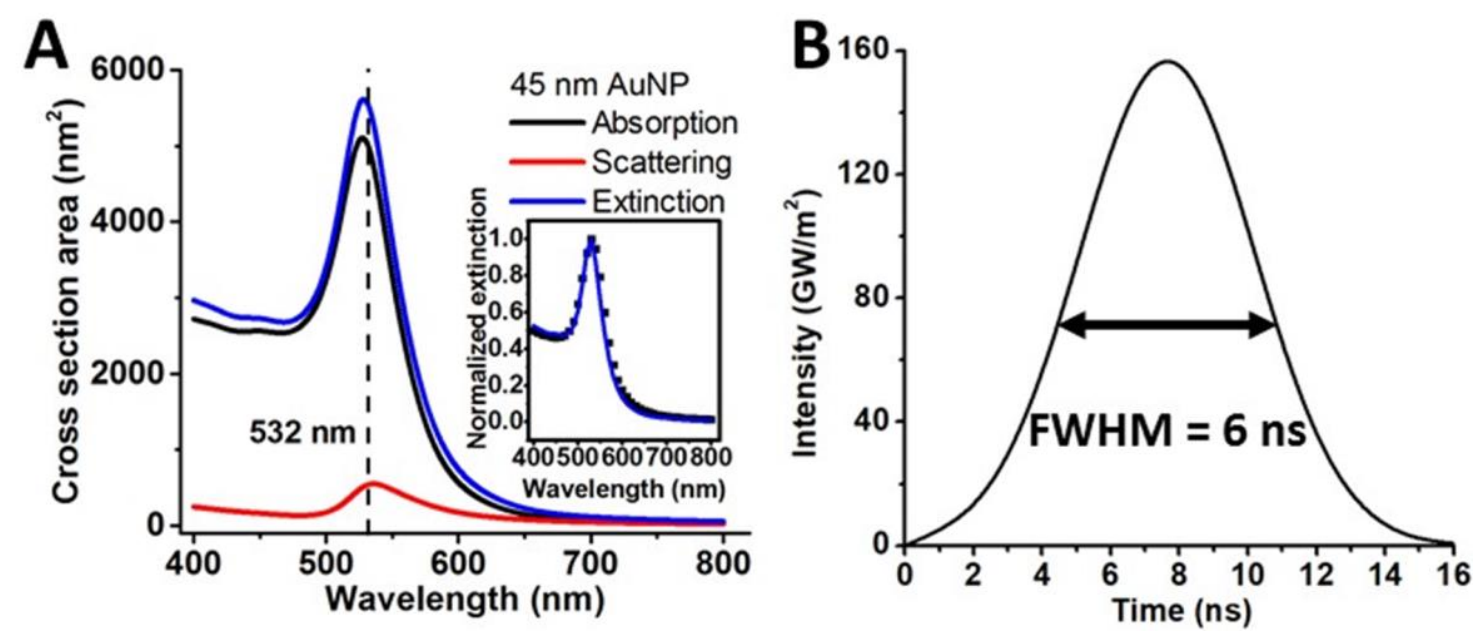

Figure S10. Optical properties of $45 \mathrm{~nm}$ AuNPs and laser power profile A) Optical properties of $45 \mathrm{~nm}$ AuNPs simulated by Mie theory. The insert shows a comparison of normalized extinction coefficient from Mie theory (line) with experimental measurement (black square). B) Nanosecond laser pulse profile (full width half maximum or FWHM $=6 \mathrm{~ns}$ ). 

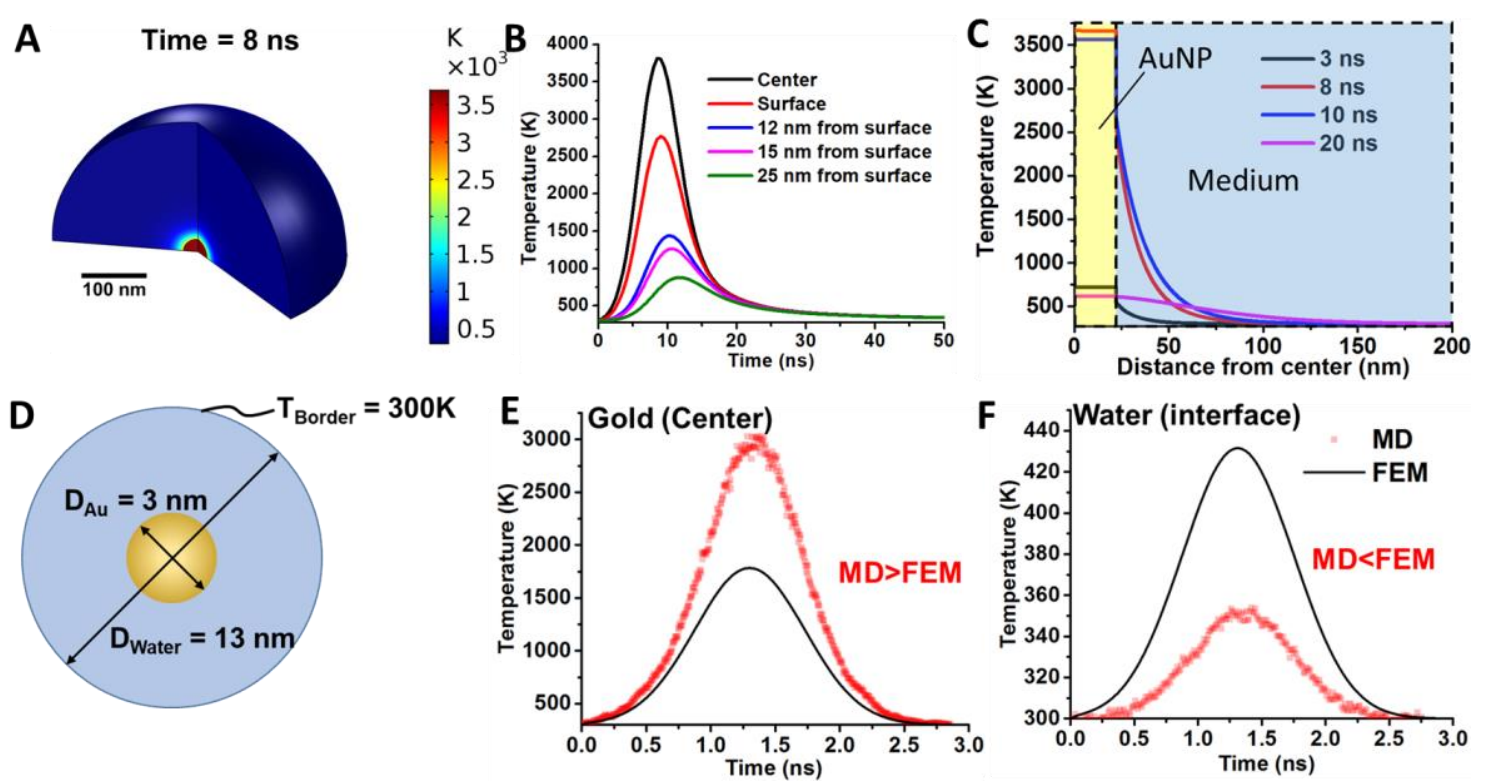

Figure S11. Simulation of gold nanoparticle laser heating and comparison of finite element method (FEM) and molecular dynamics (MD) simulation. A) 3D temperature distribution of AuNP and surrounding medium at $8 \mathrm{~ns}$ in response to a $100 \mathrm{~mJ} / \mathrm{cm}^{2}$ nanosecond laser pulse. B) Temperature distribution of AuNP and surrounding medium at different time points. C) Temperature profile of AuNP and surrounding medium as a function of time at different distances. D) Model geometry for molecular dynamics (MD) and finite element method (FEM) simulation. E) Temperature profile at the AuNP center for MD and FEM methods. F) Temperature profile of water at the gold nanoparticle surface for MD and FEM methods. 
Table S1. Heat transfer model, boundary and initial conditions.

\begin{tabular}{|c|l|}
\hline$\rho_{A u} c_{A u} \frac{\partial T}{\partial t}=\frac{1}{r^{2}} \frac{\partial}{\partial r}\left(k_{A u} r^{2} \frac{\partial T}{\partial r}\right)+G_{v}$ & (S1) \\
\hline$\rho_{\text {water }} c_{\text {water }} \frac{\partial T}{\partial t}=\frac{1}{r^{2}} \frac{\partial}{\partial r}\left(k_{\text {water }} r^{2} \frac{\partial T}{\partial r}\right)$ & (S2) \\
\hline$-\left.k_{A u} \frac{\partial T}{\partial r}\right|_{r=R}=\left.h_{g w}\left(T_{A u}-T_{\text {water }}\right)\right|_{r=R}=-\left.k_{\text {water }} \frac{\partial T}{\partial r}\right|_{r=R}$ & (S3) \\
\hline$\left.\frac{\partial T}{\partial r}\right|_{r=0}=0$ & (S4) \\
\hline$\left.T\right|_{r=10 R}=T_{r e f}=300 K$ & (S5) \\
\hline$\left.T\right|_{t=0}=T_{r e f}=300 K$ & (S6) \\
\hline$G_{v}=\frac{C_{a b s} F}{V_{N P}} \frac{2.355}{\tau \sqrt{2 \pi}} e^{-\frac{5.546\left(t-t_{0}\right)^{2}}{2 \tau^{2}}}$ & (S7) \\
\hline
\end{tabular}

Table S2. Parameters of heat transfer model.

\begin{tabular}{|c|c|c|}
\hline Parameters & Expression & Value \\
\hline Specific heat of gold & $\mathrm{c}_{\mathrm{Au}}$ & $129[\mathrm{~J} /(\mathrm{kg} \cdot \mathrm{K})]$ \\
\hline Specific heat of water & $\mathrm{c}_{\text {water }}$ & $\begin{array}{c}12010.147-80.407 \cdot \mathrm{T}+0.310 \cdot \mathrm{T}^{2}-5.382 \times 10^{-4} \cdot \mathrm{T}^{3}+3.625 \\
\mathrm{X} 10^{-7} \cdot \mathrm{T}^{4}[\mathrm{~J} /(\mathrm{kg} \cdot \mathrm{K})]^{1}\end{array}$ \\
\hline Thermal conductivity of gold & $\mathrm{k}_{\text {Au }}$ & $317[\mathrm{~W} /(\mathrm{m} \cdot \mathrm{K})]$ \\
\hline Thermal conductivity of water & $\mathrm{k}_{\text {water }}$ & $\begin{array}{c}-0.869+0.009 \cdot \mathrm{T}-1.583 \mathrm{X} 10^{-5} \cdot \mathrm{T}^{2}+7.975 \mathrm{X} 10^{-9} \cdot \mathrm{T}^{3} \\
{[\mathrm{~W} /(\mathrm{m} \cdot \mathrm{K})]^{1}}\end{array}$ \\
\hline Gold density & $\rho_{\text {Au }}$ & $19300\left[\mathrm{~kg} / \mathrm{m}^{3}\right]$ \\
\hline Water density & $\rho_{\text {water }}$ & $838.466+1.401 \cdot \mathrm{T}-0.003 \cdot \mathrm{T}^{2}+3.718 \mathrm{X} 10^{-7} \cdot \mathrm{T}^{3}\left[\mathrm{~kg} / \mathrm{m}^{3}\right]^{1}$ \\
\hline Thermal Interface conductance & $\mathrm{h}_{\mathrm{gw}}$ & $\begin{array}{c}105(\text { for } 45 \mathrm{~nm} \mathrm{AuNP})^{2} \& 60(\text { for } 3 \mathrm{~nm} \mathrm{AuNP}) \\
{\left[\mathrm{MW} /\left(\mathrm{m}^{2} \cdot \mathrm{K}\right)\right]}\end{array}$ \\
\hline
\end{tabular}

Table S3. Lennard-Jones (LJ) parameters for TIP3P and and non-bonded interactions

\begin{tabular}{|l|l|}
\hline Parameters & Values \\
\hline$\varepsilon_{A u O}(\mathrm{~kJ} / \mathrm{mol})$ & 0.588 \\
\hline$\sigma_{A u O}(\dot{A})$ & 3.383 \\
\hline$\varepsilon_{O O}(\mathrm{~kJ} / \mathrm{mol})$ & 0.646 \\
\hline$\sigma_{O O}(\dot{A})$ & 3.166 \\
\hline$\varepsilon_{O H}(\mathrm{~kJ} / \mathrm{mol})$ & 0.000 \\
\hline$\sigma_{O H}(\dot{A})$ & 2.473 \\
\hline$\varepsilon_{H H}(\mathrm{~kJ} / \mathrm{mol})$ & 0.000 \\
\hline$\sigma_{H H}(\dot{A})$ & 1.781 \\
\hline
\end{tabular}




\section{References}

(1) Wagner, W.; Kretzschmar, H.-J., International Steam Tables-Properties of Water and Steam Based on the Industrial Formulation IAPWS-IF97. Springer-Verlag: Berlin/Heidelberg, 2008.

(2) Plech, A.; Kotaidis, V.; Gresillon, S.; Dahmen, C.; von Plessen, G., Laser-Induced Heating and Melting of Gold Nanoparticles Studied by Time-Resolved X-Ray Scattering. Phys. Rev. B 2004, 70, 195423. 